\title{
Universal Critical Behavior of the Quantum Hall Plateau Transition
}

Crossover from the nonuniversal scaling regime to the universal scaling regime in quantum Hall plateau transitions Wanli Li, J. S. Xia, C. L. Vicente, N. S. Sullivan, W. Pan, D. C. Tsui, L. N. Pfeiffer, and K. W. West, Phys. Rev. B 81, $033305(2010)$

Scaling in Plateau-to-Plateau Transition: A Direct Connection of Quantum Hall Systems with the Anderson Localization Model

ibid Phys. Rev. Lett. 102, 216801 (2009).

\section{Recommended with a Commentary by Steve Kivelson, Stanford University}

Quantum Hall plateau transitions, in many ways, are the most conveniently realizable laboratory systems for studying quantum critical phenomena in a disordered system; the semiconductor devices in question are among the best characterized of all solid state systems, the magnitude and character of the disorder can be varied in predictable ways by varying details of the device construction, and one can tune through the critical point continuously by varying the magnetic field or the electron concentration (i.e. the gate voltage in appropriate devices). Enormous effort, both experimental and theoretical, was devoted to this paradigmatic problem in the 80's and 90's, but fundamental issues of perspective remain unsettled. However, to obtain clearer information from experiment, new technologies were needed to expand the dynamical range available for a scaling analysis.

In this context, it is very exciting that two new papers[1] by the Tsui group at Princeton University report the first substantial advances on this problem to emerge in a long while. These papers report studies of the $\sigma_{x y}=4 e^{2} / h \rightarrow$ $\sigma_{x y}=3 e^{2} / h$ quantum Hall plateaux transition in $\mathrm{Al}_{x} \mathrm{Ga}_{1-x}$ As quantum well devices. What is new in these studies is: A) The effect of $\mathrm{Al}$ concentration, $x$ (which, presumably, controls the magnitude of the short-range correlated disorder in the system) on the apparent critical exponents has been systematically explored. B) The studies have been carried out down to temperatures that are a factor of 20 lower than those of previous generations of studies, much expanding the dynamical range for extracting critical exponents. The principle results can be summarized as follows: 1) The width in magnetic field of the transition region between plateaus at fixed temperature obeys a scaling form

$$
\Delta B \sim\left[T^{e f f}\right]^{\kappa} ; \quad T^{e f f}=\max \left[T, T^{\star}\right]
$$

where $\Delta B$ is defined in terms of the logarithmic derivative of $\sigma_{x y}$ at the critical field, $\sigma_{x y}\left(B_{c}, T\right)=n+1 / 2$,

$$
\left.\frac{1}{\Delta B} \equiv \frac{d \log \left[\sigma_{x y}(B, T)\right]}{d B}\right|_{B=B_{c}},
$$

and $T^{\star}$ depends on the width of the device as

$$
T^{\star} \sim L^{-y}
$$

2) While the apparent value of the exponent $\kappa$ varies at moderate temperatures depending on the $\mathrm{Al}$ concentration, $x$, at low enough temperatures it approaches a "universal" value, $\kappa=0.42 \pm 0.01$. Likewise, it is found that the exponent $y=1$. (No error bars are quoted for the exponent $y$.) 3) For $x=0.85$, where we are told that the short-range correlated "alloy disorder" dominates over the long-range correlated disorder due to remote, charged impurities, $\kappa$ assumes its asymptotic low temperature value over the entire relevant range (two full decades) of $T$, while for smaller values of $x$, a crossover is observed from a larger high temperature value of $\kappa$ to a low temperature universal value.

The interpretation of observation 3) proposed by the authors is simple, plausible, and significant: So long as the critical correlation length, $\xi$, is large compared to the correlation length of the disorder, $\xi_{d i s}$, the disorder is effectively short-range correlated - since assuming a quantum critical point exists, $\xi$ must diverge as $B \rightarrow B_{c}$ and $T \rightarrow 0$, this means that the low temperature critical behavior must be universally equivalent to the case of short-range correlated disorder. However, if $\xi_{\text {dis }}$ is substantial, there will be a crossover as a function of $T$ from a different "high temperature" region where the system can be viewed as an inhomogenous mixture of regions of the two proximate quantum Hall phases, to a universal low temperature quantum critical regime.

While certain general implications of the first two observations are also clear, there are basic issues of perspective that are not, primarily because the theory of the plateau transitions is still incomplete. Specifically, the authors refer to two different theoretical perspectives which, while they have some features in common, are ultimately distinct:

1) Anderson Localization: The theoretical perspective which is favored by the authors treats the plateau transition as an Anderson transition, in which it is assumed that electron interactions play a negligible role. While no 
analytic solution of this problem has been attained, because it is a non-interacting problem much that has not been derived analytically has been computed by numerical simulations.[2-6] Here, we know that there is, at $T=0$, a sharp quantum transition at a critical field from one integer plateau to another. The localization length diverges at the critical field with a correlation length exponent, $\xi \sim\left|B-B_{c}\right|^{-\nu}$. To the extent that interactions can be ignored, the density of states at the Fermi energy is an analytic function of $\left(B-B_{c}\right)$, so there are no thermodynamic singularities at the plateau transition. Numerical studies also suggest $[2,5]$ that the $T=0$ conductivity tensor at $B=B_{c}$ is universal: at the critical field for the transition from the $n$ to $n+1$ plateau, $\sigma_{x y}\left(B_{c}, T=0\right)=(n+1 / 2) e^{2} / h$ and $\sigma_{x x}\left(B_{c}, T=0\right)=(1 / 2) e^{2} / h$.

There is a small, but very significant degree of quantitative uncertainty concerning the value of $\nu$. Calculations for electrons in the lowest Landau level with uncorrelated disorder[3] have obtained the value of $\nu=2.35 \pm 0.03$. Calculations for a slightly simpler network-model[4], until recently, produced results[5] that agreed with these within the stated error, $\nu=2.4 \pm 0.2$. However, in the last year, a more sophisticated calculation[6] has yielded a result with a considerably smaller error bar, $\nu=2.593[2.587,2.598]$. This result agrees within the error bars with earlier results on the same network model, but disagrees, well outside the error bars, with the results obtained on the potential scattering model. As it is unlikely that the critical exponents depend on the microscopic differences between the two models, the most likely conclusion is that the uncertainty in at least one of these calculations has been underestimated.

One intrinsic shortcoming of the non-interacting approach is that it gives unphysical results for non-zero $T$ unless additional dynamical couplings are introduced to augment the analysis. This problem, at core, reflects the fact that there is no mechanism within the model by which the system can relax to equilibrium. Taking a page from the theory of weak-localization, what is frequently done to "fix" this shortcoming, is to invoke a finite "phase-breaking length," $L_{\phi} \sim \alpha T^{-p / 2}$, where $\alpha$ represents the strength of the coupling of the electrons to a phase-breaking heat-bath, and $p / 2$ reflects the spectral density of the heat bath. The finite temperature behavior of the physical system is then assumed to be equivalent to that of the non-interacting model defined in a finite size system with $L \sim L_{\phi}$. Here $p$ is not a critical exponent, but a characteristic of the heat-bath, and so it is always an integer. Another shortcoming of the non-interacting description is that it cannot be straightforwardly applied to any transition involving fractional quantum Hall plateaus, where the quasiparticles have fractional statistics, and hence can never be considered as non-interacting.

2) Interacting Quantum Critical Point: An alternate view[7] of the plateau transition is that it is a true quantum critical point, and so describable by a $2+1$ dimensional quantum field theory. In classical thermodynamics, the dynamics and thermodynamics depend on entirely distinct properties of the system, but in quantum systems, the dynamics and thermodynamics are inextricably linked, due to the Heisenberg uncertainty principle, so the (imaginary) time dynamics are intrinsic to the physics, not an afterthought. A useful observation, here, is that a plateau transition (either involving integer or fractional plateaus) can be equated[8], at the expense of introducing a Chern-Simons "statistical gauge" degree of freedom, to a superconductor to insulator transition of composite bosons. Unfortunately, this problem also has not been solved, and it has not even been amenable to numerical study. However, on the basis of rather general arguments we know that the model also exhibits a sharp quantum phase transitions between neighboring plateaus with a universal critical value of the conductivity tensor at criticality. Unlike the non-interacting model, both the finite temperature and finite frequency, $\omega$, behavior of the system should be contained in the critical theory. Thus, in addition to the universal correlation length exponent, $\nu$, there is also a universal dynamical exponent, $z$, such that the thermal rounding of the phase transition obeys the scaling relation $\Delta B \sim T^{1 / \nu z}$. Moreover, if fluctuation effects are treated at gaussian (RPA) level, it has been shown that the same universal critical exponents and related values of the critical conductivities pertain to transitions involving fractional quantum Hall transitions, as well. It is not currently known, however, whether this "superuniversality" is valid beyond this level of approximation.

It has been plausibly argued[9] that the presence of Coulomb interactions implies that $z=1$ i.e. that the characteristic energy scale near criticality is $\hbar \omega \sim e^{2} / \xi$. There are also intuitive but untested arguments,[5] which suggest that the value of the localization length exponent, $\nu$, is close to its value in the non-interacting limit.

Choosing interpretations: While there is not space, here, for an in-depth discussion of the relative merits of these two interpretations, I feel there are strong reasons to prefer the quantum critical interpretation: 1) If the recent calculations[6] of $\nu$ for the non-interacting model are accepted, and if both the theoretical and experimental error bars are to be believed, then the non-interacting model yields a wrong value of $\kappa_{\text {theory }}=0.386 \pm 0.001$. 2) There is considerable evidence of superuniversality, in that the same universal critical exponents have been seen [10, 12] for both integer and fractional quantum Hall transitions, and even[13] (under appropriate circumstances) for the magnetic field driven superconductor to insulator transition - this is a natural[8] (although not established) expectation from the quantum critical perspective, and highly unnatural from the single-particle perspective. 3) There is preliminary evidence[11] of $\hbar \omega / k_{B} T$ scaling at criticality from microwave experiments, which again is evidence in favor of the 
quantum critical perspective.

[1] Wanli Li, J. S. Xia, C. Vicente, N. S. Sullivan, W. Pan, D. C. Tsui, L. N. Pfeiffer, and K. W. West, Phys. Rev. B 81, 033305 (2010); ibid Phys. Rev. Lett. 102, 216801 (2009). See, also, Wanli Li, Princeton University Thesis (2009).

[2] Y. Huo, R. E. Hertzel, and R. N. Bhatt, Phys. Rev. Lett. 70, 481 (1993).

[3] B. Huckestein, Rev. Mod. Phys. 67, 357 (1995).

[4] J. T. Chalker and P. D. Coddington, J. Phys. C 21, 2665 (1988).

[5] D. H. Lee, Z. Wang, and S. A. Kivelson, Phys. Rev. Lett. 70, 4130 (1993).

[6] K. Slevin and T. Ohtsuki, Phys. Rev. B 80, 041304 (2009)

[7] S. L. Sondhi, S. M. Girvin, J. P. Carini, and D.Shahar, Rev. Mod. Phys. 69, 315 (1997).

[8] S. Kivelson, D-H Lee, and S-C. Zhang, PRB 46, 2223 (1992).

[9] S. L. Sondhi and S. A. Kivelson, rejected (believe it or not) from the Brief Reports section of Phys. Rev. B. (1990).

[10] L. W. Wong, H W. Jiang, N. Trivedi, and E. Palm, Phys. Rev. B 51, 18033 (1995).

[11] L. W. Engel, D. Shahar, C. Kurdak, D. C. Tsui, ... Phys. Rev. Lett. 71, 2638 (1993).

[12] L. Engel, H. P. Wei, D. C. Tsui, M. Shayegan, Surf. Sci. 229, 13 (1990).

[13] M. A. Steiner, N. P. Breznay, and A. Kapitulnik, Phys. Rev. B 77, 212501 (2008). 